\title{
Postoperative Endophthalmitis following Cataract Surgery in Asia
}

\author{
Jin A Choi ${ }^{1}$ and Sung Kun Chung ${ }^{2}$ \\ ${ }^{1}$ Department of Ophthalmology and Visual Science, St. Vincent Hospital, College of Medicine, The Catholic University of Korea, \\ Seoul, Republic of Korea \\ ${ }^{2}$ Department of Ophthalmology and Visual Science, St. Mary's Hospital, College of Medicine, The Catholic University of Korea, \\ Seoul, Republic of Korea \\ Correspondence should be addressed to Sung Kun Chung, eyedoc@catholic.ac.kr
}

Received 7 October 2011; Accepted 26 November 2011

Academic Editor: R. A. Costa

Copyright (๑) 2011 J. A. Choi and S. K. Chung. This is an open access article distributed under the Creative Commons Attribution License, which permits unrestricted use, distribution, and reproduction in any medium, provided the original work is properly cited.

\begin{abstract}
Postoperative endophthalmitis is a rare clinical occurrence. However, it remains one of the most serious complications following cataract surgery because of its poor prognosis. We investigated the epidemiologic trends in postoperative endophthalmitis following cataract surgery, particularly in Asian populations. The incidence of postcataract endophthalmitis was generally consistent with epidemiologic data reported from Caucasian populations. The most frequently occurring causative organism was coagulase-negative Staphylococci in most studies of Asian populations. However, Pseudomonas aeruginosa and Nocardia were found to be the most common microorganisms in several studies. The rates of culture positivity were slightly lower than in Caucasian studies. In the evaluation of risk factors of poor visual outcomes, initial visual acuity and virulence of the causative microorganisms were generally found to be the most important risk factors. A history of pars plana vitrectomy was found to be the major risk factors for developing endophthalmitis in several studies.
\end{abstract}

\section{Introduction}

Postoperative endophthalmitis is the most devastating complication after intraocular surgery, which is commonly associated with a poor prognosis [1]. Postoperative endophthalmitis can occur following any ocular surgery in which the globe is penetrated. However, $90 \%$ of postoperative endophthalmitis occurs following cataract surgery, because cataract surgery is one of the most frequently performed intraocular surgeries in the world [2]. Fortunately, postoperative endophthalmitis after intraocular surgery is a rare clinical occurrence, but it often causes severe visual impairment or even the loss of an eye [3]. For advances in sophisticated technique and technology, cataract surgery in developed nations is regarded as a minor procedure with a short recovery time and good results $[4,5]$. With this high level of expectation, postoperative endophthalmitis after cataract surgery is especially damaging, because it can lead to permanent vision loss and loss of the eye in severe cases.
Although there are many studies on the diagnosis and treatment guidelines for postcataract endophthalmitis, most of these studies have been conducted in Western countries [ 1 , $4-14]$. Therefore, further research is needed on the variation in postoperative endophthalmitis in other countries. In this paper, we investigated the incidence, causative organism, clinical outcomes, and prognostic factors of acute postoperative endophthalmitis after cataract surgery, especially in the Asian population.

\section{Incidence of Postoperative Endophthalmitis following Cataract Surgery}

The incidence of postcataract surgery endophthalmitis varies, ranging from $<0.05 \%$ to $>0.3 \%$ [8-16]. This range in the incidence of infection appears to be consistent across numerous patient populations from all over the world [5]. In a study of ten-year incidence of endophthalmitis rate 
at Bascom Palmer Eye Institute (1984-1994) [17], the incidence of postcataract surgery endophthalmitis was $0.09 \%$. In a meta-analysis of and Taban et al. [13], the overall incidence rate of postoperative endophthalmitis was $0.128 \%$ from 1963 to 2003 . However, the incidence of postoperative endophthalmitis has changed over time and has increased to $0.265 \%$ /year over the last few decades, which coincides temporally with the development of self-sealing clear corneal incisions. Several retrospective, comparative, case-controlled studies found a significantly higher endophthalmitis rate associated with clear corneal incisions compared to sclera tunnel incisions [18-21]. Recently, Nagaki et al. [22] reported a statistically increased risk with clear corneal incisions $(0.29 \%)$ compared to sclerocorneal incisions $(0.05 \%)$.

In the Asian population, in a prospective case series which described the incidence of acute endophthalmitis in Singapore from 1996 to 2001, the authors reported an average annual incidence as $0.076 \%$ [15]. The incidence rate was relatively lower compared to previous studies, possibly because the study population included large proportions of extracapsular cataract extraction (ECCE) cases. Lalwani et al. [23] investigated postcataract endophthalmitis from 2002 to 2004 in India and reported the average 2-year annual incidence to be $0.05 \%$. In Korea, Kim et al. reported the average incidence of postoperative endophthalmitis following cataract surgery from 1987 to 1994 as $0.23 \%$ [24]. However, in a more recent study [25], where the average incidence rate was retrospectively investigated at a single center from 2000 to 2007 , it was reported as $0.359 \%$, which was in accordance with the recent increase in the incidence of postcataract endophthalmitis [13].

\section{Causative Organism}

Results in the ESCRS postoperative endophthalmitis study, the Endophthalmitis Vitrectomy Study (EVS) [4], and other studies assessing the causative organism demonstrate that Gram-positive organisms account for $90 \%$ or more of pathogens isolated in culture-positive cases of postoperative endophthalmitis following cataract surgery, with coagulase-negative Staphylococci (i.e., Staphylococcus epidermidis) and Staphylococcus aureus representing the leading causes $[10,26-28]$. In detail, approximately $70 \%$ of patients with positive cultures are infected with coagulase negative microorganisms (mostly Staphylococcus epidermidis), 10\% with Staphylococcus aureus, 9\% with Streptococcus species, 2\% with Enterococcus, 3\% with other Gram-positive species, and finally, 6\% with Gram-negative species [26, 29, 30]. In the EVS study, Gram-positive, coagulase-negative micrococci were found to cause less severe infections compared to more virulent Gram-negative, and other Gram-positive organisms, and more virulent organisms caused signs and symptoms of endophthalmitis to appear earlier $[4,6]$.

Table 1 shows the most commonly occurring microorganisms in culture-positive cases in Asia. Likewise in Caucasians, coagulase-negative Staphylococci were also found to be the most commonly found isolates [15, 25, 31-33]. However, there were several studies in which Pseudomonas species were the most common causative organism [24, 34] (Table 1). In reports from India $[35,36]$, Pseudomonas species and fungi were the most common organisms associated with postoperative endophthalmitis. Uniquely, from a study in India [37], Nocardia was found to be the most commonly occurring of all isolates tested. These differences from Asian reports may reflect geographic variations in the causative organism of postcataract endophthalmitis.

In the EVS study, $69.3 \%$ of cases demonstrated confirmed microbiologic growth from intraocular specimens [26]. In a more recent study, the culture-positive rate was $66.4 \%$ in 250 cases of endophthalmitis referred to a centre in the Netherlands [38]. In Asian study, the culture positive rate was slightly lower than in the Caucasian studies. The rate of culture positivity was $61.8 \%$ in Singapore and $52.6 \%$ in the India study. In Korea, the culture-positive rate varied from $33.3 \%$ to $75 \%$ (Table 1 ) $[24,25,31-34,39,40]$.

\section{Clinical Outcomes}

In the EVS study [4] in 1995, $86 \%$ of subjects had an initial acuity of less than 5/200, whereas $26 \%$ were light perception only. Three months later, $41 \%$ achieved 20/40, and $69 \%$ had better than $20 / 100$ vision. Nine months later, $53 \%$ achieved $20 / 40$, and $74 \%$ were better than $20 / 100$. The most common reason for the loss of visual acuity in the EVS group was attributed to macular lesion, such as epiretinal membrane, macular edema, pigmentary degeneration, and ischemia $[4,7]$. In recent studies investigating 250 cases of postcataract endophthalmitis [38], 51.6\% had final visual acuities of $20 / 40$ or more at the last examination. In the study of Lalwani et al. [23] from 1996 to $2005,49.3 \%$ of the patients showed visual acuities of $20 / 40$ or more.

In Asian populations, the proportions of patients who showed final visual acuities of $20 / 40$ or more were $29.41 \%$ in India [37] and 50.5\% in Singapore [15]. In Korea, the proportion varied from $22.2 \%$ to $83.3 \%$ (Table 2) [24, $25,31-34,39,40]$, and the proportion of patients who underwent pars plana vitrectomy ( $\mathrm{ppV}$ ) was somewhat larger (23.5 to $93.9 \%$ ) compared to the studies in Singapore (29.4\%) and India (21.1\%) (Table 2). Variable initial visual acuities at the time of diagnosis as well as uneven proportion of $\mathrm{ppV}$ may explain these variable results in the final visual acuities in Korean studies [24, 25, 31-34, 39, 40].

\section{Risk Factors}

In the EVS study [4], multiple independent risk factors associated with poor visual outcomes were found. The strongest risk factor was light perception only vision. Additional risk factors included old age, diabetes, corneal infiltrate or ring ulcer, compromised porterior capsule, low or high intraocular pressure, afferent pupillary defect, rubeosis, and an absent red reflex [4, 41]. Moreover, infecting organisms played an important role in visual prognosis. They reported that a final visual acuity of 20/100 was achieved with Grampositive, coagulase-negative micrococci $84 \%$ of the time, followed by S. aureus, 50\%, Streptococci, 30\%, Enterococci, 
TABLE 1: Causative organisms found in postcataract surgery endophthalmitis in Asia.

\begin{tabular}{|c|c|c|c|c|}
\hline Study & Years included & Organism detection rate & Most common isolates & Location \\
\hline \multirow{4}{*}{ Kim et al. [25] } & \multirow{4}{*}{$2000-2007$} & \multirow{4}{*}{$47.4 \%$} & S. epidermidis (67\%) & \multirow{4}{*}{ Korea } \\
\hline & & & S. aureus $(11 \%)$ & \\
\hline & & & Enterococcus (11\%) & \\
\hline & & & S. pneumoniae (11\%) & \\
\hline \multirow{5}{*}{ Jung et al. [31] } & \multirow{5}{*}{ 2001-2006 } & \multirow{5}{*}{$50.9 \%$} & S. epidermitis (28\%) & \multirow{5}{*}{ Korea } \\
\hline & & & Streptococcus sp (28\%) & \\
\hline & & & Enterobacter sp (17\%) & \\
\hline & & & P. aeruginosa (17\%) & \\
\hline & & & Fungus $(10 \%)$ & \\
\hline \multirow{6}{*}{ Yi et al. [32] } & \multirow{6}{*}{ 1993-1996 } & \multirow{6}{*}{$55.6 \%$} & S. epidermidis (20\%) & \multirow{6}{*}{ Korea } \\
\hline & & & S. aureus $(10 \%)$ & \\
\hline & & & S. viridence $(10 \%)$ & \\
\hline & & & P. aeruginosa $(10 \%)$ & \\
\hline & & & Serratoa marcescens (10\%) & \\
\hline & & & Fungus $(30 \%)$ & \\
\hline \multirow{2}{*}{ Choi et al. [34] } & \multirow{2}{*}{ 1992-1995 } & \multirow{2}{*}{$75.0 \%$} & Pseudomonas sp (50\%) & \multirow{2}{*}{ Korea } \\
\hline & & & S. epidermidis (42\%) & \\
\hline Kim et al. [24] & 1987-1994 & $\begin{array}{c}33.3 \% \text { (aqueous humor) } \\
100 \% \text { (vitreous tap) }\end{array}$ & $\begin{array}{l}\text { Pseudomonas sp }(50 \%) \\
\text { Serratis marcescens }(33 \%) \\
\text { S. pneumoniae }(17 \%)\end{array}$ & Korea \\
\hline \multirow{5}{*}{ Wong and chee [15] } & \multirow{5}{*}{$1996-2001$} & \multirow{5}{*}{$61.8 \%$} & S. epidermidis (57\%) & \multirow{5}{*}{ Singapore } \\
\hline & & & Streptococcus sp (14\%) & \\
\hline & & & S. aureus $(10 \%)$ & \\
\hline & & & P. aeruginosa (5\%) & \\
\hline & & & Other GNB (14\%) & \\
\hline \multirow{2}{*}{ Lalitha et al. [37] } & \multirow{2}{*}{$2002-2003$} & \multirow{2}{*}{$52.6 \%$} & Nocardia $(60 \%)$ & \multirow{2}{*}{ India } \\
\hline & & & S. epidermidis (40\%) & \\
\hline
\end{tabular}

TABLE 2: Visual acuity outcomes after postcataract surgery endophthalmitis in Asia.

\begin{tabular}{|c|c|c|c|c|c|}
\hline Study & Years included & $\begin{array}{l}\text { Visual acuity at the time of diagnosis } \\
\qquad(\% \text { of } \geq 20 / 200)\end{array}$ & $\mathrm{ppV} \%$ & $\begin{array}{c}\text { Visual acuity at final } \\
\text { followup } \\
(\% \text { of } \geq 20 / 200)\end{array}$ & $\begin{array}{c}\text { Visual acuity at final } \\
\text { followup } \\
(\% \text { of } \geq 20 / 40)\end{array}$ \\
\hline Kim et al. [25] & $2000-2007$ & $34.5 \%$ & $55.1 \%$ & $62.1 \%$ & $34.5 \%$ \\
\hline Jung et al. [31] & 2001-2006 & $21.6 \%$ & $52.3 \%$ & $77.0 \%$ & $49.2 \%$ \\
\hline Yi et al. [32] & 1993-2006 & $0.0 \%$ & $66.7 \%$ & $50.0 \%$ & $27.8 \%$ \\
\hline Choi et al. [34] & 1992-2005 & $0.0 \%$ & $31.2 \%$ & $75 \%$ & $50.0 \%$ \\
\hline Kim et al. [24] & 1987-1994 & $33.3 \%$ & $33.3 \%$ & $100.0 \%$ & $83.3 \%$ \\
\hline Park et al.* [33] & 1989-1993 & $3.1 \%$ & $93.8 \%$ & $46.9 \%$ & $25.0 \%$ \\
\hline Wong and chee. [15] & $1996-2001$ & $35.3 \%$ & $29.4 \%$ & $64.7 \%$ & $47.1 \%$ \\
\hline Lalitha et al. [37] & $2002-2003$ & $21.1 \%$ & $21.1 \%$ & $31.6 \%$ & $26.3 \%$ \\
\hline
\end{tabular}

${ }^{*}$ This study includes cases with infectious endophthalmitis including traumatic, endogenous, and posttrabeculectomy cases as well as postcataract endophthalmitis.

14\%, and Gram-negative organisms, 56\% [26]. In accordance with these findings, Lalwani et al. and Pijl et al. also emphasized the treatment outcome after endophthalmitis was found to be highly dependent on the causative organism although the outcomes for Gram-negative bacteria and
S. aureus were better than previously reported $[23,38]$. It is reported that some bacteria, such as $S$. epidermidis, may sterilize spontaneously during the ocular inflammatory response [42]. Also, in culture-negative cases of postoperative endophthalmitis, there may be cases with noninfectious 
inflammation. These may explain the strong effect of virulence of microorganisms on the outcomes of postoperative endophthalmitis.

In the Asian population, the phacoemulsification technique and intraoperative posterior capsule rupture were associated with a 1.8 times higher risk of acute culturepositive endophthalmitis in Singapore [15]. Interestingly, in India, the ECCE technique was found to be the major risk factor for developing endophthalmitis [37].

In Korea, the infecting organism was found to play an important role in the visual prognosis $[31,32,40]$. The final visual acuity was found to be associated with the poor initial visual acuity $[25,31]$. The history of $\mathrm{ppV}$ was associated with poor visual outcomes in several studies $[31,32,34]$. As a treatment option in endophthalmitis, $\mathrm{ppV}$ has potential benefits such as reduction of the infecting organism, toxins, inflammatory materials, and opacities as well as collection of samples for culture and potential improvement of intravitreal antibiotic distribution. The $\mathrm{ppV}$ in the treatment of endophthalmitis requires highly sophisticated surgical techniques because of corneal edema, severe anterior chamber reaction, and undilated pupil in the patients, which may limit the positive outcomes in endophthalmitis. The need for more complex equipment and an operating room for $\mathrm{ppV}$ may delay treatment compared to clinic-based procedures such as vitreous tap and biopsy, which could worsen the outcome $[1,6,7]$.

In a more recent study, Kuhn and Gini [43] reported that over $90 \%$ of the patients who underwent complete vitrectomy achieved $>20 / 40$ of final visual acuity. With modern equipment such as adjustable cutting and flow rates as well as better visualization with panoramic viewing systems, $\mathrm{ppV}$ after postoperative endophthalmitis is expected to give better outcomes compared to previous studies.

\section{Conclusions}

The incidences of postcataract endophthalmitis were generally consistent with epidemiologic data reported from Caucasian populations. The most causative organism was coagulase-negative Staphylococci in most studies carried out with the Asian population. However, Pseudomonas aeruginosa and Nocardia species were found to be the most common microorganism in several studies. The rates of culture positivity were slightly lower than in Caucasian studies. In the evaluation of risk factors of poor visual outcomes, initial visual acuity and virulence of causative microorganisms was generally found to be the most important risk factors. Studies have shown that a history of $\mathrm{ppV}$ have been found to be the major risk factors for developing endophthalmitis.

Globally, postoperative endophthalmitis remains one of the most serious complications following cataract surgery. Early diagnosis and aggressive treatment with antimicrobial therapy as well as surgical intervention is mandatory for optimal visual outcomes. Currently, EVS studies have been standard in the treatment of postcataract endophthalmitis. However, there are local variations, especially with regards to the causative organisms and risk factors in Asian countries.
Therefore, for more appropriate treatment of postoperative endophthalmitis, clinicians need to consider these differences in their practice.

\section{References}

[1] J. I. Maguire, "Postoperative endophthalmitis: optimal management and the role and timing of vitrectomy surgery," Eye, vol. 22, no. 10, pp. 1290-1300, 2008.

[2] H. Verbraeken, "Treatment of postoperative endophthalmitis," Ophthalmologica, vol. 209, no. 3, pp. 165-171, 1995.

[3] M. S. Kresloff, A. A. Castellarin, and M. A. Zarbin, "Endophthalmitis," Survey of Ophthalmology, vol. 43, no. 3, pp. 193224, 1998.

[4] "Results of the endophthalmitis vitrectomy study: a randomized trial of immediate vitrectomy and of intravenous antibiotics for the treatment of postoperative bacterial endophthalmitis," Archives of Ophthalmology, vol. 113, no. 12, pp. 1479-1496, 1995.

[5] R. E. Fintelmann and A. Naseri, "Prophylaxis of postoperative endophthalmitis following cataract surgery: current status and future directions," Drugs, vol. 70, no. 11, pp. 1395-1409, 2010.

[6] N. Mamalis, L. Kearsley, and E. Brinton, "Postoperative endophthalmitis," Current Opinion in Ophthalmology, vol. 13, no. 1, pp. 14-18, 2002.

[7] C. A. Lemley and D. P. Han, "Endophthalmitis: a review of current evaluation and management," Retina, vol. 27, no. 6, pp. 662-680, 2007.

[8] E. S. West, A. Behrens, P. J. McDonnell, J. M. Tielsch, and O. D. Schein, "The incidence of endophthalmitis after cataract surgery among the U.S. medicare population increased between 1994 and 2001," Ophthalmology, vol. 112, no. 8, pp. 1388-1394, 2005.

[9] W. V. Hatch, G. Cernat, D. Wong, R. Devenyi, and C. M. Bell, "Risk factors for acute endophthalmitis after cataract surgery: a population-based study," Ophthalmology, vol. 116, no. 3, pp. 425-430, 2009.

[10] Endophthalmitis Study Group and European Society of Cataract \& Refractive Surgeons, "Prophylaxis of postoperative endophthalmitis following cataract surgery: results of the ESCRS multicenter study and identification of risk factors," Journal of Cataract \& Refractive Surgery, vol. 33, pp. 978-988, 2007.

[11] R. D. Ravindran, R. Venkatesh, D. F. Chang, S. Sengupta, J. Gyatsho, and B. Talwar, "Incidence of post-cataract endophthalmitis at Aravind Eye Hospital. Outcomes of more than 42,000 consecutive cases using standardized sterilization and prophylaxis protocols," Journal of Cataract and Refractive Surgery, vol. 35, no. 4, pp. 629-636, 2009.

[12] H. S. Al-Mezaine, D. Kangave, A. Al-Assiri, and A. A. AlRajhi, "Acute-onset nosocomial endophthalmitis after cataract surgery. Incidence, clinical features, causative organisms, and visual outcomes," Journal of Cataract and Refractive Surgery, vol. 35, no. 4, pp. 643-649, 2009.

[13] M. Taban, A. Behrens, R. L. Newcomb et al., "Acute endophthalmitis following cataract surgery: a systematic review of the literature," Archives of Ophthalmology, vol. 123, no. 5, pp. 613620, 2005.

[14] Z. I. Carrim, J. Richardson, and W. N. Wykes, "Incidence and visual outcome of acute endophthalmitis after cataract surgery-the experience of an eye department in Scotland," British Journal of Ophthalmology, vol. 93, no. 6, pp. 721-725, 2009. 
[15] T. Y. Wong and S. P. Chee, "The epidemiology of acute endophthalmitis after cataract surgery in an Asian population," Ophthalmology, vol. 111, no. 4, pp. 699-705, 2004.

[16] V. A. Deramo, J. C. Lai, D. M. Fastenberg, and I. J. Udell, "Acute endophthalmitis in eyes treated prophylactically with gatifloxacin and moxifloxacin," American Journal of Ophthalmology, vol. 142, no. 5, pp. 721.e1-725.e1, 2006.

[17] T. M. Aaberg, H. W. Flynn, J. Schiffman, and J. Newton, "Nosocomial acute-onset postoperative endophthalmitis survey: a 10-year review of incidence and outcomes," Ophthalmology, vol. 105, no. 6, pp. 1004-1010, 1998.

[18] B. A. Cooper, N. M. Holekamp, G. Bohigian, and P. A. Thompson, "Case-control study of endophthalmitis after cataract surgery comparing scleral tunnel and clear corneal wounds," American Journal of Ophthalmology, vol. 136, no. 2, pp. 300305, 2003.

[19] S. Lertsumitkul, P. C. Myers, M. T. O’Rourke, and J. Chandra, "Endophthalmitis in the western Sydney region: a case-control study," Clinical and Experimental Ophthalmology, vol. 29, no. 6, pp. 400-405, 2001.

[20] P. J. McDonnell, E. D. Donnenfeld, H. D. Perry et al., "New horizons in fluoroquinolone therapy," Ophthalmology Times, vol. 27, supplement 1, pp. 1-15, 2002.

[21] D. Barrow, M. McDermott, D. Elliot, and R. Frank, "Acute postoperative endophthalmitis and modern cataract surgery technique [ARVO abstract 1340]," Investigative Ophthalmology \& Visual Science, vol. 42, p. S24, 2001.

[22] Y. Nagaki, S. Hayasaka, C. Kadoi et al., "Bacterial endophthalmitis after small-incision cataract surgery: effect of incision placement and intraocular lens type," Journal of Cataract and Refractive Surgery, vol. 29, no. 1, pp. 20-26, 2003.

[23] G. A. Lalwani, H. W. Flynn Jr., I. U. Scott et al., "Acute-onset endophthalmitis after clear corneal cataract surgery (19962005). Clinical features, causative organisms, and visual acuity outcomes," Ophthalmology, vol. 115, no. 3, pp. 473-476, 2008.

[24] J. M. Kim, I. W. Park, and H. B. Lee, "Endophthalmitis after intraocular surgery," The Journal of Korean Ophthalmological Society, vol. 35, pp. 1451-1456, 1994.

[25] W. J. Kim, E. Y. Kweon, D. W. Lee, and N. C. Choi, "Postoperative endophthalmitis following cataract surgery over an eight-year period," Korean Journal of Ophthalmology, vol. 49, pp. 1771-1778, 2008.

[26] D. P. Han, S. R. Wisniewski, L. A. Wilson et al., "Spectrum and susceptibilities of microbiologic isolates in the Endophthalmitis Vitrectomy Study; the Endophthalmitis Vitrectomy Study Group," American Journal of Ophthalmology, vol. 122, pp. 117, 1996, correction, 920.

[27] M. S. Benz, I. U. Scott, H. W. Flynn Jr., N. Unonius, and D. Miller, "Endophthalmitis isolates and antibiotic sensitivities: a 6-year review of culture-proven cases," American Journal of Ophthalmology, vol. 137, no. 1, pp. 38-42, 2004.

[28] F. M. Recchia, B. G. Busbee, R. B. Pearlman, C. A. CarvalhoRecchia, and A. C. Ho, "Changing trends in the microbiologic aspects of postcataract endophthalmitis," Archives of Ophthalmology, vol. 123, no. 3, pp. 341-346, 2005.

[29] B. H. Doft, "The Endophthalmitis Vitrectomy Study," Archives of Ophthalmology, vol. 109, no. 4, pp. 487-489, 1991.

[30] A. Pinna, L. A. Sechi, S. Zanetti, D. Delogu, and F. Carta, "Adherence of ocular isolates of Staphylococcus epidermidis to ACRYSOF intraocular lenses: a scanning electron microscopy and molecular biology study," Ophthalmology, vol. 107, no. 12, pp. 2162-2166, 2000.

[31] J. Y. Jung, B. Y. Ko, and B. Y. Kim, "Factors associated with a poor visual results in acute endophthalmitis after cataract surgery," The Journal of the Korean Ophthalmological Society, vol. 49, pp. 1242-1247, 2008.

[32] W. M. Yi, N. H. Kwak, M. H. Kim et al., "Postoperative endophthalmitis following intraocular surgery," Journal of the Korean Ophthalmological Society, vol. 39, pp. 2095-2102, 1998.

[33] K. S. Park, Y. G. Park, W. K. Min, and B. H. Ahn, "Microbiological diagnosis and visual outcome of infectious endophthalmitis," Journal of the Korean Ophthalmological Society, vol. 35, pp. 1715-1722, 1994.

[34] G. J. Choi, G. J. Yoon, and K. S. Na, "Clinical observation on postoperative endophthalmitis following cataract surgery," Journal of the Korean Ophthalmological Society, vol. 37, pp. 961-966, 1996.

[35] A. R. Anand, K. L. Therese, and H. N. Madhavan, "Spectrum of aetiological agents of postoperative endophthalmitis and antibiotic susceptibility of bacterial isolates," Indian Journal of Ophthalmology, vol. 48, no. 2, pp. 123-128, 2000.

[36] A. Gupta, V. Gupta, A. Gupta et al., "Spectrum and clinical profile of post cataract surgery endophthalmitis in North India," Indian Journal of Ophthalmology, vol. 51, no. 2, pp. 139-145, 2003.

[37] P. Lalitha, J. Rajagopalan, K. Prakash, K. Ramasamy, N. V. Prajna, and M. Srinivasan, "Postcataract endophthalmitis in South India: incidence and outcome," Ophthalmology, vol. 112, no. 11, pp. 1885-1890, 2005.

[38] B. J. Pijl, T. Theelen, M. A. D. Tilanus, R. Rentenaar, and N. Crama, "Acute endophthalmitis after cataract surgery: 250 consecutive cases treated at a tertiary referral center in the Netherlands," American Journal of Ophthalmology, vol. 149, no. 3, pp. 482.e2-487.e2, 2010.

[39] S. B. Lee, J. W. Han, S. K. Chung, and N. H. Baek, "Factors associated with visual outcomes of postoperative endophthalmitis following cataract surgery," Journal of the Korean Ophthalmological Society, vol. 46, pp. 1618-1623, 2005.

[40] S. E. Chung and D. I. Ham, "Visual prognosis of cultureproven bacterial endophthalmitis," Journal of the Korean Ophthalmological Society, vol. 47, pp. 1292-1297, 2006.

[41] B. H. Doft, S. F. Kelsey, and S. R. Wisniewski, "Additional procedures after the initial vitrectomy or tap-biopsy in the endophthalmitis vitrectomy study," Ophthalmology, vol. 105, no. 4, pp. 707-716, 1998.

[42] T. A. Meredith, A. Trabelsi, M. J. Miller, E. Aguilar, and L. A. Wilson, "Spontaneous sterilization in experimental Staphylococcus epidermidis endophthalmitis," Investigative Ophthalmology and Visual Science, vol. 31, no. 1, pp. 181-186, 1990.

[43] F. Kuhn and G. Gini, "Ten years after. Are findings of the endophthalmitis vitrectomy study still relevant today?" Graefe's Archive for Clinical and Experimental Ophthalmology, vol. 243, no. 12, pp. 1197-1199, 2005. 


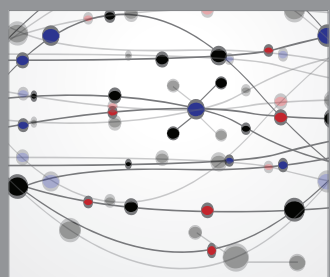

The Scientific World Journal
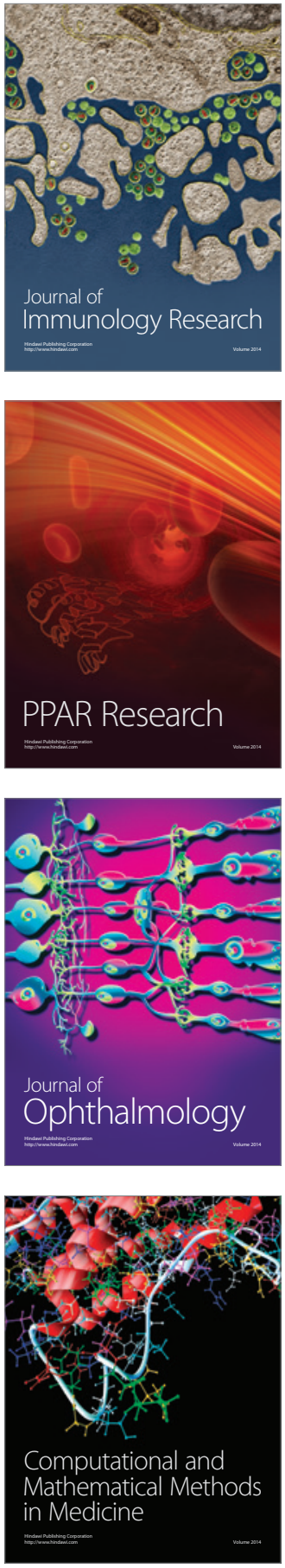

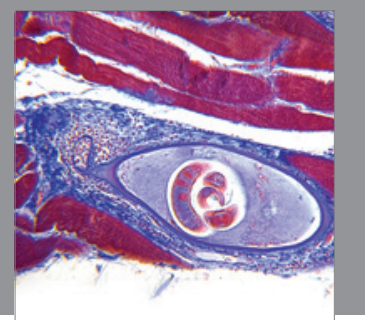

Gastroenterology

Research and Practice
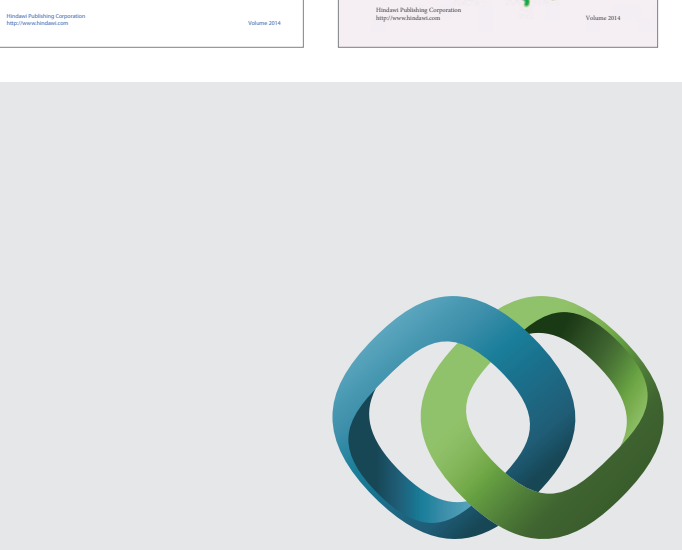

\section{Hindawi}

Submit your manuscripts at

http://www.hindawi.com
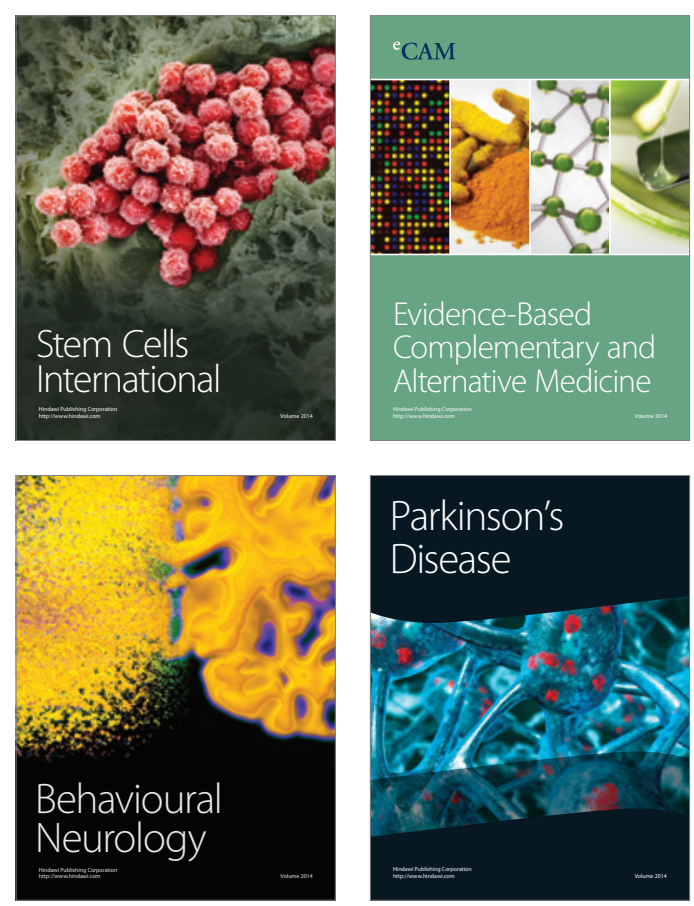

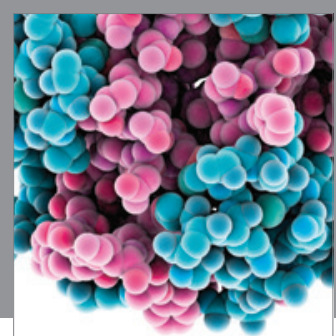

Journal of
Diabetes Research

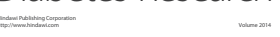

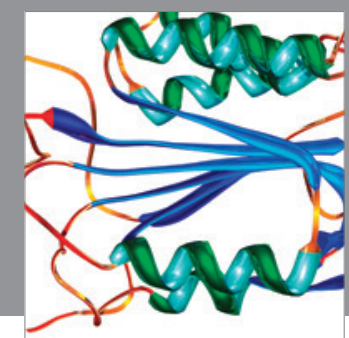

Disease Markers
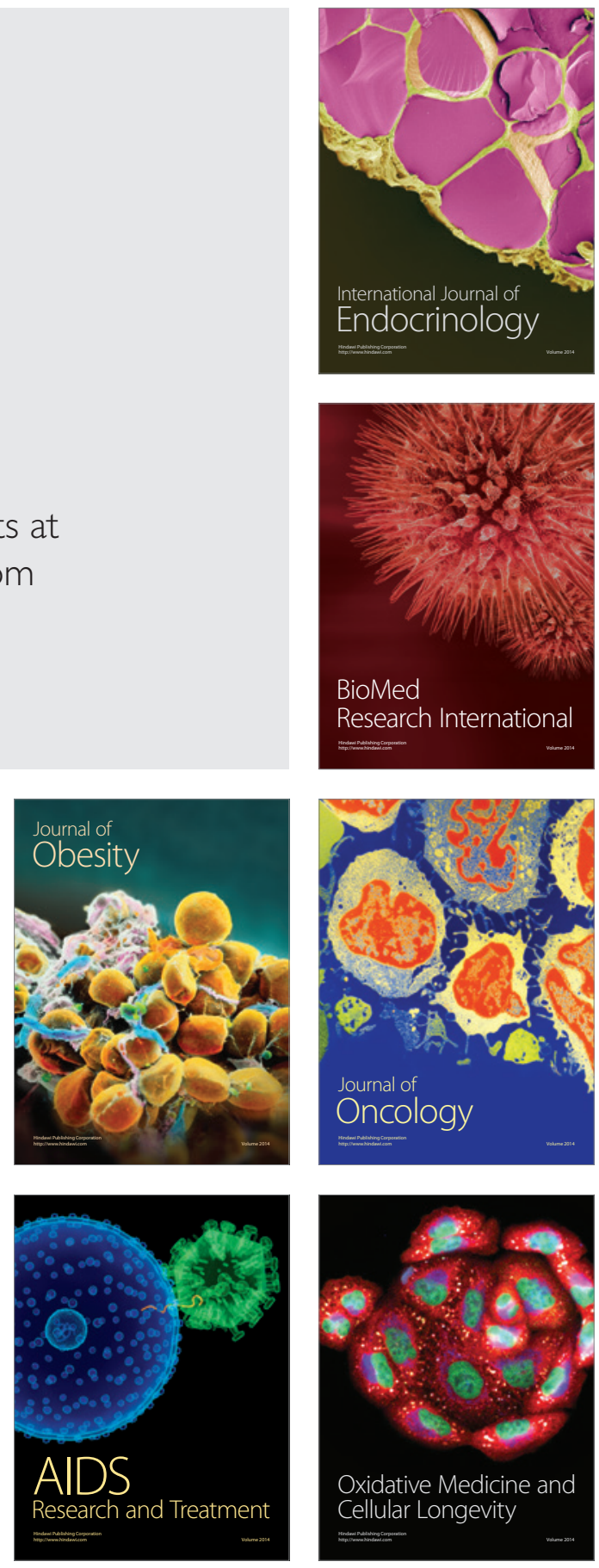